\title{
Systematic Design of Complex Orthogonal Space-Time Block Codes with High Rates
}

\author{
Weifeng Su \\ Department of ECE \\ University of Maryland, \\ College Park, MD 20742, USA \\ Email: weifeng@eng.umd.edu
}

\author{
Xiang-Gen Xia \\ Department of ECE \\ University of Delaware, \\ Newark, DE 19716 USA \\ Email: xxia@ee.udel.edu
}

\author{
K. J. Ray Liu \\ Department of ECE \\ University of Maryland, \\ College Park, MD 20742, USA \\ Email: kjrliu@eng.umd.edu
}

\begin{abstract}
In this paper, we develop a systematic design method to generate high-rate space-time block codes (STBCs) from complex orthogonal designs for any number of transmit antennas. The resulting complex orthogonal STBCs have the best known rates, which are conjectured "optimal". Two constructions with rates $2 / 3$ and $5 / 8$ are further illustrated for 6 and 7 transmit antennas, respectively.
\end{abstract}

\section{INTRODUCTION}

Space-time block codes (STBCs) from complex orthogonal designs have received extensive interest recently, see for example [1]-[10]. The special structure of orthogonal designs guarantees that these codes achieve full diversity and have a very simple maximum-likelihood (ML) decoding algorithm. The transmitted symbols can be decoded separately, not jointly, at receiver.

A complex orthogonal design (COD) in variables $x_{1}, x_{2}, \cdots, x_{k}$ is a $p \times n$ matrix $G$ such that: i) the entries of $G$ are complex linear combinations of $x_{1}, x_{2}, \cdots, x_{k}$ and their complex conjugates $x_{1}^{*}, x_{2}^{*}, \cdots, x_{k}^{*}$; and ii) the columns of $G$ are orthogonal to each other, i.e.,

$$
G^{\mathcal{H}} G=\left(\left|x_{1}\right|^{2}+\left|x_{2}\right|^{2}+\cdots+\left|x_{k}\right|^{2}\right) I_{n \times n},
$$

where $I_{n}$ is the $n \times n$ identity matrix, and the superscript $\mathcal{H}$ stands for the complex conjugate and transpose of a matrix. The rate of $G$ is defined as $R=k / p$. This design $G$ can be used to generate a STBC for $n$ transmit antennas. The resulting STBC has a rate $k / p$, which means that each codeword with block length $p$ carries $k$ information symbols. See [2] for more details about the encoding scheme and the fast decoding algorithm.

The first STBC from COD was proposed by Alamouti [1] for 2 transmit antennas, which is due to the following $2 \times 2$ design with two complex variables:

$$
G_{2}=\left[\begin{array}{rr}
x_{1} & x_{2} \\
-x_{2}^{*} & x_{1}^{*}
\end{array}\right] .
$$

Clearly, the rate of $G_{2}$ is 1 . For $n=4$ transmit antennas, there are CODs of rate $R=3 / 4$ [2], [3], [4], [5], for example,

$$
G_{4}=\left[\begin{array}{rrrr}
x_{1} & x_{2} & x_{3} & 0 \\
-x_{2}^{*} & x_{1}^{*} & 0 & x_{3} \\
-x_{3}^{*} & 0 & x_{1}^{*} & -x_{2} \\
0 & -x_{3}^{*} & x_{2}^{*} & x_{1}
\end{array}\right] .
$$

TABLE I

COMPLEX ORTHOGONAL SPACE-TIME BLOCK CODE WITH RATE $2 / 3$ FOR SIX TRANSMIT ANTENNAS

$$
\left[\begin{array}{rrrrrr}
x_{1} & x_{2} & x_{3} & 0 & x_{7} & 0 \\
-x_{2}^{*} & x_{1}^{*} & 0 & x_{4}^{*} & 0 & x_{11}^{*} \\
-x_{3}^{*} & 0 & x_{1}^{*} & x_{5}^{*} & 0 & x_{12}^{*} \\
0 & -x_{3}^{*} & x_{2}^{*} & x_{6}^{*} & 0 & x_{13}^{*} \\
0 & -x_{4} & -x_{5} & x_{1} & x_{8} & 0 \\
x_{4} & 0 & -x_{6} & x_{2} & x_{9} & 0 \\
x_{5} & x_{6} & 0 & x_{3} & x_{10} & 0 \\
-x_{6}^{*} & x_{5}^{*} & -x_{4}^{*} & 0 & 0 & x_{14}^{*} \\
-x_{7}^{*} & 0 & 0 & -x_{8}^{*} & x_{1}^{*} & x_{15}^{*} \\
0 & -x_{7}^{*} & 0 & -x_{9}^{*} & x_{2}^{*} & x_{16}^{*} \\
0 & 0 & -x_{7}^{*} & -x_{10}^{*} & x_{3}^{*} & x_{17}^{*} \\
-x_{9}^{*} & x_{8}^{*} & 0 & 0 & x_{4}^{*} & x_{18}^{*} \\
-x_{10}^{*} & 0 & x_{8}^{*} & 0 & x_{5}^{*} & x_{19}^{*} \\
0 & -x_{10}^{*} & x_{9}^{*} & 0 & x_{6}^{*} & x_{20}^{*} \\
x_{8} & x_{9} & x_{10} & -x_{7} & 0 & 0 \\
0 & -x_{11} & -x_{12} & 0 & -x_{15} & x_{1} \\
x_{11} & 0 & -x_{13} & 0 & -x_{16} & x_{2} \\
x_{12} & x_{13} & 0 & 0 & -x_{17} & x_{3} \\
0 & 0 & x_{14} & -x_{11} & -x_{18} & x_{4} \\
0 & -x_{14} & 0 & -x_{12} & -x_{19} & x_{5} \\
x_{14} & 0 & 0 & -x_{13} & -x_{20} & x_{6} \\
x_{15} & x_{16} & x_{17} & 0 & 0 & x_{7} \\
0 & -x_{18} & -x_{19} & x_{15} & 0 & x_{8} \\
x_{18} & 0 & -x_{20} & x_{16} & 0 & x_{9} \\
x_{19} & x_{20} & 0 & x_{17} & 0 & x_{10} \\
-x_{13}^{*} & x_{12}^{*} & -x_{11}^{*} & -x_{14}^{*} & 0 & 0 \\
-x_{16}^{*} & x_{15}^{*} & 0 & x_{18}^{*} & -x_{11}^{*} & 0 \\
-x_{17}^{*} & 0 & x_{15}^{*} & x_{19}^{*} & -x_{12}^{*} & 0 \\
0 & -x_{17}^{*} & x_{16}^{*} & x_{20}^{*} & -x_{13}^{*} & 0 \\
x_{20}^{*} & -x_{19}^{*} & x_{18}^{*} & 0 & x_{14}^{*} & 0 \\
& & & & &
\end{array}\right.
$$

The design for $n=3$ transmit antennas is obtained simply by taking the first three columns of $G_{4}$. A class of CODs with rate $R=1 / 2$ was given by Tarokh, Jafarkhani, and Calderbank in [2] by taking advantage of the full-rate real orthogonal designs [2], [12]. Later in [6], two generalized CODs with rates $7 / 11$ and $3 / 5$ were constructed for $n=5$ and $n=6$ transmit antennas, respectively. Recently, a COD with rate $2 / 3$ was presented for $n=5$ transmit antennas [8].

In case of CODs of square size $(p=n)$, it has been shown in [2], [3] that $4 \times 4$ CODs of rate 1 do not exist, and a general result was given in [5] that $R \leq(a+1) /\left(2^{a} b\right)$ if $n=2^{a} b, b$ odd, which is related to the Hurwitz theory [11], [13]. In case of CODs of non-square size $(p \geq n)$, it was proved in [9] that 
rate 1 cannot be achieved for $n \geq 3$ transmit antennas. Later in [7], we showed that for CODs without linear processing, the rate cannot be greater than $3 / 4$ for $n \geq 3$ transmit antennas. Further in [10], it was showed that this result holds for CODs with linear processing.

In this paper, we propose a systematic design method to generate high-rate complex orthogonal STBCs for any number of transmit antennas. The obtained designs have the best known rates. Moreover, these designs indicate that CODs with non-square size do provide larger rates than those with square size.

\section{Construct High-Rate CODs for Any Number of TRANSMIT ANTENNAS}

At first, we will present two hand-crafted high-rate CODs for $n=6$ and $n=7$ transmit antennas explicitly. Then we will describe a basic design methodology and provide a general algorithm to generate high-rate CODs for any number of transmit antennas.

For $n=6$ transmit antennas, we construct a $30 \times 6 \mathrm{COD}$ $G_{6}$ with rate $R=2 / 3$ in Table I. This design contains $k=20$ symbols and has a block length or delay $p=30$. For $n=7$ transmit antennas, a $56 \times 7 \mathrm{COD} G_{7}$ is specified in Table II. This design contains $k=35$ symbols and has a block length $p=56$. Clearly, the rate of $G_{7}$ is $R=k / p=5 / 8$.

The basic methodology of constructing the orthogonal designs $G_{6}$ and $G_{7}$ is stated as follows. For convenience, let's say a row in an orthogonal design is conjugate (nonconjugate) if all symbols except zeros in this row have (do not have) complex conjugate. For example, the first row of the orthogonal design $G_{2}$ in (2) is non-conjugate, and the second row is conjugate. We started from $G_{1}=x_{1} I_{1}$, and generated $G_{m}$ from $G_{m-1}$ iteratively for any $2 \leq m \leq n$. In each iteration from $G_{m-1}$ to $G_{m}$, we added a new column with some new symbols. The number of the new symbols and the position of each new symbol in the new column depend on the numbers of conjugate rows and non-conjugate rows in $G_{m-1}$. If the number of non-conjugate rows is not less than that of conjugate rows, we add some new symbols into the non-conjugate rows and set some zeros in the conjugate rows. If the number of non-conjugate rows is less than that of conjugate rows, we put some new symbols with complex conjugate into the conjugate rows and set some zeros in the non-conjugate rows accordingly. Due to the new symbols in the $m$-th column, we have to arrange some additional rows to guarantee the orthogonality, i.e., all the $m$ columns should be orthogonal to each other.

With the basic ideas described above, we have developed a general algorithm as follows. Denote $k_{m-1}$ and $p_{m-1}$, respectively, the numbers of symbols and rows in $G_{m-1}$ for any $m \geq 2$.

- Initialization: Start from $G_{1}=x_{1} I_{1}$.

- Generate $G_{m}$ from $G_{m-1}$ : for $m=2,3, \cdots, n$

1 Calculate $\nu_{0}$ and $\nu_{1}$, the numbers of non-conjugate rows and conjugate rows in $G_{m-1}$, respectively.

TABLE II

COMPLEX ORTHOGONAL SPACE-TIME BLOCK CODE WITH RATE $5 / 8$ FOR SEVEN TRANSMIT ANTENNAS

\begin{tabular}{|c|c|c|c|c|c|c|}
\hline$x_{1}$ & $x_{2}$ & $x_{3}$ & 0 & $x_{7}$ & 0 & $x_{21}$ \\
\hline$-x_{2}^{*}$ & $x_{1}^{*}$ & 0 & $x_{4}^{*}$ & 0 & $x_{11}^{*}$ & 0 \\
\hline$-x_{3}^{*}$ & 0 & $x_{1}^{*}$ & $x_{5}^{*}$ & 0 & $x_{12}^{*}$ & 0 \\
\hline 0 & $-x_{3}^{*}$ & $x_{2}^{*}$ & $x_{6}^{*}$ & 0 & $x_{13}^{*}$ & 0 \\
\hline 0 & $-x_{4}$ & $-x_{5}$ & $x_{1}$ & $x_{8}$ & 0 & $x_{22}$ \\
\hline$x_{4}$ & 0 & $-x_{6}$ & $x_{2}$ & $x_{9}$ & 0 & $x_{23}$ \\
\hline$x_{5}$ & $x_{6}$ & 0 & $x_{3}$ & $x_{10}$ & 0 & $x_{24}$ \\
\hline$-x_{6}^{*}$ & $x_{5}^{*}$ & $-x_{4}^{*}$ & 0 & 0 & $x_{14}^{*}$ & 0 \\
\hline$-x_{7}^{*}$ & 0 & 0 & $-x_{8}^{*}$ & $x_{1}^{*}$ & $x_{15}^{*}$ & 0 \\
\hline 0 & $-x_{7}^{*}$ & 0 & $-x_{9}^{*}$ & $x_{2}^{*}$ & $x_{16}^{*}$ & 0 \\
\hline 0 & 0 & $-x_{7}^{*}$ & $-x_{10}^{*}$ & $x_{3}^{*}$ & $x_{17}^{*}$ & 0 \\
\hline$-x_{9}^{*}$ & $x_{8}^{*}$ & 0 & 0 & $x_{4}^{*}$ & $x_{18}^{*}$ & 0 \\
\hline$-x_{10}^{*}$ & 0 & $x_{8}^{*}$ & 0 & $x_{5}^{*}$ & $x_{19}^{* 0}$ & 0 \\
\hline & $-x_{10}^{*}$ & $x_{9}^{*}$ & 0 & $x_{6}^{*}$ & $x_{20}^{*}$ & 0 \\
\hline$x_{8}$ & $x_{9}$ & $x_{10}$ & $-x_{7}$ & 0 & 0 & $x_{25}$ \\
\hline 0 & $-x_{11}$ & $-x_{12}$ & 0 & $-x_{15}$ & $x_{1}$ & $x_{26}$ \\
\hline$x_{11}$ & 0 & $-x_{13}$ & 0 & $-x_{16}$ & $x_{2}$ & $x_{27}$ \\
\hline$x_{12}$ & $x_{13}$ & 0 & 0 & $-x_{17}$ & $x_{3}$ & $x_{28}$ \\
\hline 0 & 0 & $x_{14}$ & $-x_{11}$ & $-x_{18}$ & $x_{4}$ & $x_{29}$ \\
\hline 0 & $-x_{14}$ & 0 & $-x_{12}$ & $-x_{19}$ & $x_{5}$ & $x_{30}$ \\
\hline$x_{14}$ & 0 & 0 & $-x_{13}$ & $-x_{20}$ & $x_{6}$ & $x_{31}$ \\
\hline$x_{15}$ & $x_{16}$ & $x_{17}$ & 0 & 0 & $x_{7}$ & $x_{32}$ \\
\hline 0 & $-x_{18}$ & $-x_{19}$ & $x_{15}$ & 0 & $x_{8}$ & $x_{33}$ \\
\hline$x_{18}$ & 0 & $-x_{20}$ & $x_{16}$ & 0 & $x_{9}$ & $x_{34}$ \\
\hline$x_{19}$ & $x_{20}$ & 0 & $x_{17}$ & 0 & $x_{10}$ & $x_{35}$ \\
\hline$-x_{13}^{*}$ & $x_{12}^{*}$ & $-x_{11}^{*}$ & $-x_{14}^{*}$ & 0 & 0 & 0 \\
\hline$-x_{16}^{*}$ & $x_{15}^{*}$ & 0 & $x_{18}^{*}$ & $-x_{11}^{*}$ & 0 & 0 \\
\hline$-x_{17}^{*}$ & & $x_{15}^{*}$ & $x_{19}^{*}$ & $-x_{12}^{*}$ & 0 & 0 \\
\hline 0 & $-x_{17}^{*}$ & $x_{16}^{*}$ & $x_{20}^{*}$ & $-x_{13}^{*}$ & 0 & 0 \\
\hline$x_{20}^{*}$ & $-x_{19}^{*}$ & $x_{18}^{*}$ & 0 & $x_{14}^{*}$ & 0 & 0 \\
\hline$-x_{21}^{*}$ & & 0 & $-x_{22}^{*}$ & 0 & $-x_{26}^{*}$ & $x_{1}^{*}$ \\
\hline & $-x_{21}^{*}$ & 0 & $-x_{23}^{*}$ & 0 & $-x_{27}^{*}$ & $x_{2}^{*}$ \\
\hline 0 & & $-x_{21}^{*}$ & $-x_{24}^{*}$ & 0 & $-x_{28}^{*}$ & $x_{3}^{*}$ \\
\hline$-x_{23}^{*}$ & $x_{22}^{*}$ & 0 & 0 & 0 & $-x_{29}^{*}$ & $x_{4}^{*}$ \\
\hline$-x_{24}^{*}$ & & $x_{22}^{*}$ & 0 & 0 & $-x_{30}^{*}$ & $x_{5}^{*}$ \\
\hline & $-x_{24}^{*}$ & $x_{23}^{*}$ & 0 & 0 & $-x_{31}^{*}$ & $x_{6}^{*}$ \\
\hline 0 & 0 & 0 & $x_{25}^{*}$ & $-x_{21}^{*}$ & $-x_{32}^{*}$ & $x_{7}^{*}$ \\
\hline$-x_{25}^{*}$ & 0 & 0 & 0 & $-x_{22}^{*}$ & $-x_{33}^{*}$ & $x_{8}^{*}$ \\
\hline & $-x_{25}^{*}$ & 0 & 0 & $-x_{23}^{*}$ & $-x_{34}^{*}$ & $x_{9}^{*}$ \\
\hline & 0 & $-x_{25}^{*}$ & 0 & $-x_{24}^{*}$ & $-x_{35}^{*}$ & $x_{10}^{*}$ \\
\hline$-x_{27}^{*}$ & $x_{26}^{*}$ & 0 & $x_{29}^{*}$ & 0 & 0 & $x_{11}^{*}$ \\
\hline$-x_{28}^{*}$ & 0 & $x_{26}^{*}$ & $x_{30}^{*}$ & 0 & 0 & $x_{12}^{*}$ \\
\hline 0 & $-x_{28}^{*}$ & $x_{27}^{*}$ & $x_{31}^{*}$ & 0 & 0 & $x_{13}^{*}$ \\
\hline$-x_{31}^{*}$ & $x_{30}^{*}$ & $-x_{29}^{*}$ & 0 & 0 & 0 & $x_{14}^{*}$ \\
\hline$-x_{32}^{*}$ & 0 & 0 & $-x_{33}^{*}$ & $x_{26}^{*}$ & 0 & $x_{15}^{*}$ \\
\hline & $-x_{32}^{*}$ & 0 & $-x_{34}^{*}$ & $x_{27}^{*}$ & 0 & $x_{16}^{*}$ \\
\hline & 0 & $-x_{32}^{*}$ & $-x_{35}^{*}$ & $x_{28}^{*}$ & 0 & $x_{17}^{*}$ \\
\hline$-x_{34}^{*}$ & $x_{33}^{*}$ & 0 & 0 & $x_{29}^{*}$ & 0 & $x_{18}^{*}$ \\
\hline$-x_{35}^{*}$ & & $x_{33}^{*}$ & 0 & $x_{30}^{*}$ & 0 & $x_{19}^{*}$ \\
\hline 0 & $-x_{35}^{*}$ & $x_{34}^{*}$ & 0 & $x_{31}^{*}$ & 0 & $x_{20}^{*}$ \\
\hline$x_{22}$ & $x_{23}$ & $x_{24}$ & $-x_{21}$ & $-x_{25}$ & 0 & 0 \\
\hline$x_{26}$ & $x_{27}$ & $x_{28}$ & 0 & $x_{32}$ & $-x_{21}$ & 0 \\
\hline 0 & $-x_{29}$ & $-x_{30}$ & $x_{26}$ & $x_{33}$ & $-x_{22}$ & 0 \\
\hline$x_{29}$ & 0 & $-x_{31}$ & $x_{27}$ & $x_{34}$ & $-x_{23}$ & 0 \\
\hline$x_{30}$ & $x_{31}$ & 0 & $x_{28}$ & $x_{35}$ & $-x_{24}$ & 0 \\
\hline$-x_{33}$ & $-x_{34}$ & $-x_{35}$ & $x_{32}$ & 0 & $x_{25}$ & 0 \\
\hline
\end{tabular}


2 Add some new symbols into the $m$-th column from the first row to the $p_{m-1}$-th row. If $\nu_{0} \geq \nu_{1}$, then add new symbols $x_{k_{m-1}+1}, x_{k_{m-1}+2}, \cdots, x_{k_{m-1}+\nu_{0}}$ into the nonconjugate rows, and set zeros into the conjugate rows; If $\nu_{0}<\nu_{1}$, then add new symbols with complex conjugate $x_{k_{m-1}+1}^{*}, x_{k_{m-1}+2}^{*}, \cdots, x_{k_{m-1}+\nu_{1}}^{*}$ into the conjugate rows, and set zeros into the non-conjugate rows.

3 Since the symbols $x_{1}, x_{2}, \cdots, x_{k_{m-1}}$ must appear in each column, we further specify the entries in the $m$-th column after the $p_{m-1}$-th row as follows. If $\nu_{0} \geq \nu_{1}$, then put $x_{1}^{*}, x_{2}^{*}, \cdots, x_{k_{m-1}}^{*}$ into the $m$-th column from the $\left(p_{m-1}+1\right)$-th row to the $\left(p_{m-1}+k_{m-1}\right)$-th row; If $\nu_{0}<\nu_{1}$, then put $x_{1}, x_{2}, \cdots, x_{k_{m-1}}$ into the $m$-th column from the $\left(p_{m-1}+1\right)$-th row to the $\left(p_{m-1}+k_{m-1}\right)$-th row.

4 According to the orthogonality of the $m$-th column to each of the first $m-1$ columns, we fill the entries of the first $m-1$ columns from the $\left(p_{m-1}+1\right)$-th row to the $\left(p_{m-1}+k_{m-1}\right)$-th row as follows.

$$
\begin{gathered}
\text { for row }=1: p_{m-1} ; \text { for } \mathrm{col}=1: m-1 \\
\text { if } \mid G_{m-1}(\text { row }, \mathrm{col})|=| x_{i} \mid \text { for any } 1 \leq i \leq m-1, \\
\text { if } \operatorname{sign}\left(G_{m-1}(\text { row }, \text { col })\right)=1 \text {, then } \\
\quad G_{m}\left(p_{m-1}+i, \mathrm{col}\right)=-G_{m}^{*}(\text { row }, m) ; \\
\text { if } \operatorname{sign}\left(G_{m-1}(\text { row }, \text { col })\right)=-1, \text { then } \\
G_{m}\left(p_{m-1}+i, c o l\right)=G_{m}^{*}(\text { row }, m) ;
\end{gathered}
$$

end;

Set zeros to those unspecified entries;

5 After Step 4, the $m$-th column is ready. However, we need to arrange some additional rows to ensure the orthogonality of the first $m-1$ columns. In the additional rows, simply put zeros at the $m$-th column.

Set RowCount $=p_{m-1}+k_{m-1}$.

$$
\begin{aligned}
& \text { for row }=p_{m-1}+1: p_{m-1}+k_{m-1} \\
& \quad \text { if } G_{m}(\text { row }, \text { col } 1) \neq 0 \text { and } G_{m}(\text { row, col } 2) \neq 0 \text { for }
\end{aligned}
$$$$
1 \leq \operatorname{col} 1<\operatorname{col} 2 \leq m-1 \text {, then }
$$$$
\text { Set NeedNewRow }=\text { TRUE; }
$$$$
X_{11}=G_{m}(\text { row }, \text { col } 1) ; X_{12}=G_{m}(\text { row }, \text { col } 2) \text {; }
$$$$
\text { for NewRow }=p_{m-1}+k_{m-1}+1: \text { RowCount }
$$$$
X_{21}=G_{m}(\text { NewRow }, \text { coll }) \text {; }
$$$$
X_{22}=G_{m}(\text { NewRow, col2); }
$$$$
\text { if } X_{21}=0 \text { and }\left|X_{22}\right|=\left|X_{11}\right| \text {, then }
$$$$
\text { if } X_{11}=X_{22}^{*} \text {, then }
$$$$
G_{m}(\text { NewRow }, \text { coll } 1)=-X_{12}^{*}
$$$$
\text { if } X_{11}=-X_{22}^{*} \text {, then }
$$$$
G_{m}(\text { NewRow }, \text { coll } 1)=X_{12}^{*} \text {; }
$$

NeedNewRow $=$ FALSE;

if $\left|X_{21}\right|=\left|X_{12}\right|$ and $X_{22}=0$, then
TABLE III

HIGH-RATE STBCS FROM COMPLEX ORTHOGONAL DESIGNS FOR $2 \leq n \leq 18$ TRANSMIT ANTENNAS

\begin{tabular}{|l|c|c|c|}
\hline & $\begin{array}{c}\text { Symbols } \\
k\end{array}$ & $\begin{array}{c}\text { Block Length } \\
p\end{array}$ & $\begin{array}{c}\text { Rate } \\
k / p\end{array}$ \\
\hline$n=2$ & 2 & 2 & 1 \\
$n=3$ & 3 & 4 & $3 / 4$ \\
$n=4$ & 6 & 8 & $3 / 4$ \\
$n=5$ & 10 & 15 & $2 / 3$ \\
$n=6$ & 20 & 30 & $2 / 3$ \\
$n=7$ & 35 & 56 & $5 / 8$ \\
$n=8$ & 70 & 112 & $5 / 8$ \\
$n=9$ & 126 & 210 & $3 / 5$ \\
$n=10$ & 252 & 420 & $3 / 5$ \\
$n=11$ & 462 & 792 & $7 / 12$ \\
$n=12$ & 924 & 1584 & $7 / 12$ \\
$n=13$ & 1716 & 3003 & $4 / 7$ \\
$n=14$ & 3432 & 6006 & $4 / 7$ \\
$n=15$ & 6435 & 11440 & $9 / 16$ \\
$n=16$ & 12870 & 22880 & $9 / 16$ \\
$n=17$ & 24310 & 43758 & $5 / 9$ \\
$n=18$ & 48620 & 87516 & $5 / 9$ \\
\hline
\end{tabular}

$$
\begin{gathered}
\text { if } X_{21}=X_{12}^{*} \text {, then } \\
G_{m}(\text { NewRow }, \text { col } 2)=-X_{11}^{*} ; \\
\text { if } X_{21}=-X_{12}^{*}, \text { then } \\
G_{m}(\text { NewRow }, \text { col } 1)=X_{11}^{*} ; \\
\text { NeedNewRow }=\text { FALSE; } \\
\text { if }\left|X_{21}\right|=\left|X_{12}\right| \text { and }\left|X_{22}\right|=\left|X_{11}\right| \text {, then } \\
\text { NeedNewRow }=\text { FALSE; }
\end{gathered}
$$

end;

if NeedNewRow $=T R U E$, then

RowCount $=$ RowCount +1 ;

$G_{m}($ RowCount, col 1$)=-G_{m}^{*}($ row, col 2$) ;$

$G_{m}($ RowCount, $\operatorname{col} 2)=G_{m}^{*}($ row, $\operatorname{col} 1)$;

end;

- Let $k_{m}=k_{m-1}+\max \left(\nu_{0}, \nu_{1}\right), p_{m}=$ RowCount, and repeat the above iteration if $m<n$.

According to Steps 4 and 5, the orthogonality of the resulting design is guaranteed. The above general algorithm can be used to generate high-rate CODs for any number of transmit antennas. In Table III, we list the number of symbols, block length and rate of the resulting design for $n=2,3, \cdots, 18$ transmit antennas, respectively. We observe from the table that for $n \leq 18$ transmit antennas, the rate of the obtained design satisfies a rule of $\left(n_{0}+1\right) /\left(2 n_{0}\right)$ if $n=2 n_{0}$ or $n=2 n_{0}-1$, which is conjectured optimal in [10] for any $n$. We believe that the proposed algorithm will continue to generate CODs with the optimal rate for $n>18$.

\section{CONCLUSION}

We presented in this paper a systematic design method to generate high-rate complex orthogonal STBCs for any number 
of transmit antennas. Also, the designs for $n \leq 18$ transmit antennas were obtained and two designs with rates $2 / 3$ and $5 / 8$ were further illustrated for 6 and 7 transmit antennas, respectively. All of the resulting CODs have the best known rates that are conjectured optimal. Moreover, these designs indicate that CODs with non-square size do provide larger rates than those with square size. For example, for $n=8$ transmit antennas, the maximum rate of CODs with square size is $1 / 2$ [5] while the COD obtained here has a rate of $5 / 8$. This phenomenon was observed in [2] in case of real orthogonal designs.

Note that for $n>10$ transmit antennas, the block length of the obtained CODs is large. For example, for $n=16$ transmit antennas, the block length is $p=22880$, while the block length of the rate $1 / 2$ code from real orthogonal design is $p=256$ [2]. Therefore, for large number of transmit antennas, the resulting CODs are more of theoretical interest.

\section{REFERENCES}

[1] S. Alamouti, "A simple transmit diversity technique for wireless communications," IEEE J. Select. Areas Commun., vol. 16, no. 8, pp. 1451-1458, 1998.

[2] V. Tarokh, H. Jafarkhani, and A. R. Calderbank, "Space-time block codes from orthogonal designs," IEEE Trans. Inform. Theory, vol. 45, no. 5, pp. $1456-1467,1999$
[3] G. Ganesan and P. Stoica, "Space-time block codes: a maximum SNR approach," IEEE Trans. Inform. Theory, vol. 47, no. 4, pp. 1650-1656, May 2001.

[4] B. M. Hochwald, T. L. Marzetta, and C. B. Papadias, "A transmitter diversity scheme for wideband CDMA systems based on space-time spreading," IEEE J. Select. Areas Commun., vol. 19, no. 1, pp. 48-60, 2001.

[5] O. Tirkkonen and A. Hottinen, "Square-matrix embeddable space-time block codes for complex signal constellations," IEEE Trans. Inform. Theory, vol. 48, no. 2, pp. 1122-1126, Feb. 2002.

[6] W. Su and X.-G. Xia, "Two generalized complex orthogonal space-time block codes of rates $7 / 11$ and $3 / 5$ for 5 and 6 transmit antennas," IEEE Trans. Inform. Theory, vol. 49, no. 1, pp.313-316, Jan. 2003.

[7] W. Su and X.-G. Xia, "On space-time block codes from complex orthogonal designs," Wireless Personal Communications (Kluwer Academic Publishers), vol. 25, no. 1, pp.1-26, April 2003.

[8] X.-B. Liang, "A high-rate orthogonal space-time block code," IEEE Commun. Letters, vol. 7, no. 5, pp.222-223, May 2003

[9] X.-B. Liang and X.-G. Xia, "On the nonexistence of rate-one generalized complex orthogonal designs," IEEE Trans. Inform. Theory, to appear.

[10] H. Wang and X.-G. Xia, "Upper bounds of rates of space-time block codes from complex orthogonal designs," IEEE Trans. Inform. Theory, Oct. 2003, to appear.

[11] A. V. Geramita and J. M. Geramita, "Complex orthogonal designs," Journal of Combinatorial Theory, Series A (25), pp.211-225, 1978.

[12] A. V. Geramita and J. Seberry, Orthogonal Designs, Quadratic Forms and Hadamard Matrices, Lecture Notes in Pure and Applied Mathematics, vol.43, New York and Basel: Marcel Dekker, 1979.

[13] D. B. Shapiro, Compositions of Quadratic Forms, New York, De Gruyter, 2000. 